\title{
Soliton dynamics in semiconductor photonic crystals
}

Chad Husko $^{\mathrm{a}}$, Andrea Blanco-Redondo ${ }^{\mathrm{b}}$, Simon Lefrancois ${ }^{\mathrm{b}}$, Benjamin J. Eggleton ${ }^{\mathrm{b}}$, Thomas F. Krauss ${ }^{c}$, Matthias Wulf ${ }^{\mathrm{d}}$, L. Kobus Kuipers ${ }^{\mathrm{d}}$, Chee Wei Wong ${ }^{\mathrm{e}}$, Sylvain Combriéf ${ }^{\text {, Alfredo }}$ De Rossi ${ }^{\mathrm{f}}$, and Pierre Colman ${ }^{\mathrm{g}}$

${ }^{a}$ Center for Nanoscale Materials, Argonne National Laboratory, Argonne, Illinois 60439, USA ${ }^{\mathrm{b}}$ Centre for Ultrahigh bandwidth Devices for Optical Systems (CUDOS), Institute of Photonics and Optical Science (IPOS), School of Physics, The University of Sydney, NSW 2006, Australia ${ }^{\mathrm{c}}$ Department of Physics, University of York, York YO10 5DD, UK

${ }^{\mathrm{d} C e n t e r}$ for Nanophotonics, FOM Institute AMOLF, Science Park 104, 1098 XG, Amsterdam, The Netherlands

eMesoscopic Optics and Quantum Electronics Laboratory, University of California, Los Angeles, California 90095, USA

fThales Research and Technology, 1 Av. A. Fresnel 128, 91767 Palaiseau, France 'Institut d'Electronique Fontamentale (IEF), Universite Paris-Sud, Orsay, France

\begin{abstract}
Semiconductor optical waveguides have been the subject of intense study as both fundamental objects of study, as well as a path to photonic integration. In this talk I will focus on the nonlinear evolution of optical solitons in photonic crystal waveguides made of semiconductor materials. The ability to independently tune the dispersion and the nonlinearity in photonic crystal waveguides enables the examination of completely different nonlinear regimes in the same platform. I will describe experimental efforts utilizing time-resolved measurements to reveal a number of physical phenomena unique to solitons in a free carrier medium. The experiments are supported by analytic and numerical models providing a deeper insight into the physical scaling of these processes.
\end{abstract}

Keywords: photonic crystals, solitons, nonlinear optics, integrated optics, semiconductor optics, nanophotonics

\section{INTRODUCTION}

Solitons are localized waves formed by a balance of focusing and defocusing effects. These nonlinear waves exist in diverse forms of matter yet exhibit similar properties including stability, periodic recurrence, and particlelike evolution. In the optical domain a temporal soliton can form from the controlled balance of the nonlinear Kerr effect with linear group-velocity dispersion (GVD). ${ }^{1}$ In semiconductor materials one must also account for nonlinear absorption and free carriers at typical intensity levels. ${ }^{2}$ The nonlinear absorption attenuates the peak power, restricting the desirable nonlinear phase change due to the Kerr effect. In addition, the nonlinear absorption generates free carriers. The free-carrier plasma modifies the nonlinear pulse evolution with both dispersive $\left(\mathrm{FCD}, n_{F C}\right)$ and absorptive $(\mathrm{FCA}, \sigma)$ contributions leading to non-trivial dynamics unavailable in other optical systems. For example, while in the spectral domain optical pulses undergo a spectral blueshift due to $\mathrm{FCD},{ }^{3-5}$ in contrast the temporal properties are governed by the dynamic interaction of FCD and dispersion together leading to, for example, nonlinear pulse temporal broadening. ${ }^{6}$ These free-carrier effects also interplay with and modulate the classical soliton evolution. Temporal solitons in semiconductors have been shown ${ }^{7-9}$ including soliton self-frequency blueshift ${ }^{10}$ and soliton acceleration. ${ }^{11,12}$ In this talk we describe the dynamics and physical effects unique to solitons in semiconductors through experiment, theory, and numerical modeling.

Send correspondence to chusko@anl.gov 


\section{SOLITONS IN SEMICONDUCTOR PHOTONIC CRYSTAL WAVEGUIDES}

\subsection{Classic soliton regime (weakly perturbed)}

Bright solitons are one class of analytic solutions to the nonlinear Schrödinger equation (NLSE) considering SPM $(\gamma)$ and anomalous GVD $\left(\beta_{2}<0\right)$. The length scales associated with these effects are the dispersion length $L_{D}=T_{o}^{2} / \beta_{2}$, where $T_{o}=T / \Gamma, T_{F W H M}$ is the full-width half-maximum, $\Gamma=2 \cosh ^{-1}(\sqrt{2})=1.76$ for hyperbolic secant pulses, and the nonlinear length $L_{N L}=\left(\gamma P_{o}\right)^{-1}$. The soliton number

$$
N=\sqrt{L_{D} / L_{N L}}=\frac{T_{o}^{2} \gamma P_{o} S^{2}}{\beta_{2}},
$$

defines the conditions for soliton propagation. The $S^{2}$ term refers to slow-light enhancement of the Kerr nonlinearity in photonic crystal waveguides. ${ }^{13}$ The pulse propagates without dispersing as a fundamental soliton when $N=1$, while the solutions with $N>1$ correspond to higher-order solitons. In this latter case, the pulse experiences recurrent periodic evolution by first compressing, then splitting into two distinct peaks, which later re-collide, before finally resuming the initial shape after a soliton period $z_{o}=\frac{\pi}{2} L_{D}$. In addition, larger $N$ numbers lead to greater compression and evolve on shorter length scales. ${ }^{1,14}$ Fig. 1(a) summarizes the key parameters (nonlinear effects $\gamma$ and dispersion $\beta_{2}$ ) in the various platforms in which soliton propagation has been demonstrated. The red circle indicates the region of photonic crystal waveguides.

The quest to demonstrate soliton dynamics at the chip scale is challenging due to the short (millimeter or less) interaction length and compatibility of compact laser sources, the combination of which has hindered the development of an integrable soliton compressor. Periodic dielectric media are a particularly attractive platform to observe solitons on a chip scale as they can be designed for very high, very low, positive and negative dispersion values (for example, up to five orders of magnitude higher than in fibre). ${ }^{7,15}$ This fact, combined with the possibility of enhancing the nonlinear effects by using slow light, facilitates soliton evolution on sub-millimeter length scales. We now describe experiments demonstrating the temporal evolution of optical solitons in a semiconductor photonic chip using autocorrelation measurements. We input pulses with a FWHM

(a)

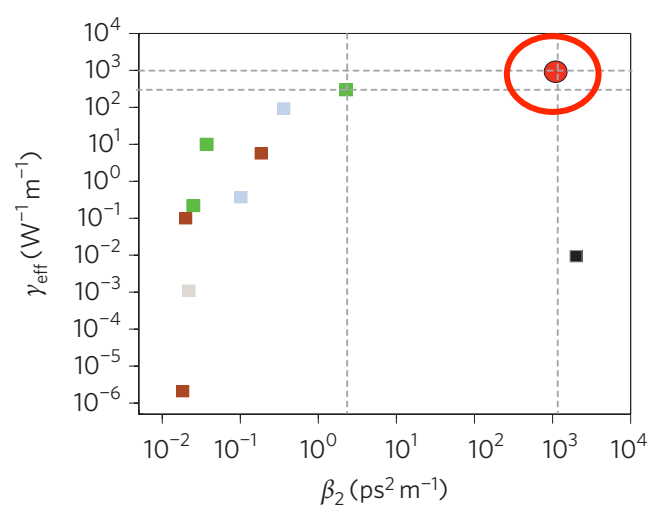

(b)

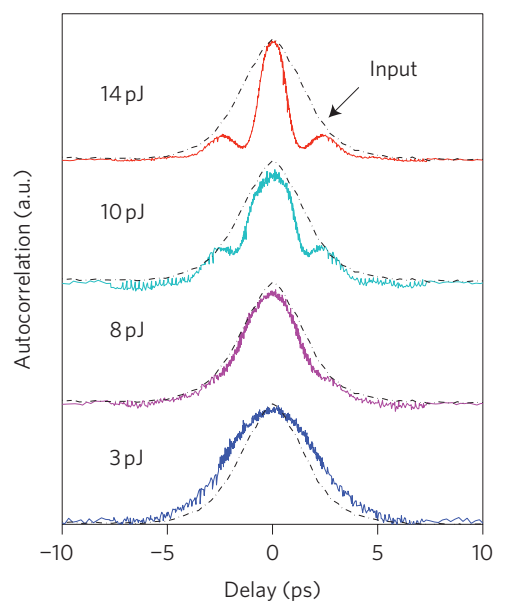

Figure 1. Temporal optical solitons in photonic crystal waveguides. (a) Nonlinear $\left(\gamma_{e f f}\right)$ and GVD $\left(\beta_{2}\right)$ parameters of different material systems with experimentally demonstrated soliton effects. The PhCWG examined in this work simultaneously exhibits both large $\gamma_{e f f}$ and GVD coefficients in a single material system enabling compression of picosecond pulses at picojoule energies (Watt peak powers) and millimeter length scales. (b) Autocorrelation measurements at 1555 $\mathrm{nm}$ with 1.8-ps exponential input pulses (dotted lines). The pulse experiences dispersion-induced broadening at small pulse energies before contracting at larger pulse energies, thereby demonstrating characteristic higher-soliton behavior. Here the material is GaInP, which exhibits suppressed two-photon absorption at this wavelength. 
duration of $1.8 \mathrm{ps}$ (decaying exponential pulse) and a spectral width of $1.2 \mathrm{~nm}$ centered at $1555 \mathrm{~nm}$, giving a dispersion length $L_{D}$ of $1.1 \mathrm{~mm}$. Under these conditions the dispersion length is shorter than the device length, $L=1.5 \mathrm{~mm}\left(L<L_{D}\right)$. Fig. 1(b) summarizes the experimental results. At the lowest pulse energies (weak nonlinear effects) we observed dispersive temporal broadening. As the pulse energy increases, the output pulse returns to the input duration before further compressing to a minimum duration of 800 fs (deconvolution factor of 1.54 for hyperbolic secant), limited only by the output power of the laser. Despite the almost triangular autocorrelation input trace (significantly different from a hyperbolic secant input), the pulse redistributes its energy into a hyperbolic secant form by the time it reaches the output, an important property of solitons. These results follow traditional soliton theory of the nonlinear Kerr effect balanced by GVD with reasonably high fidelity. This outcome was enabled by the use of the wide-gap material GaInP (1.9 eV) which suppresses nonlinear absorption at these wavelengths and intensity levels. ${ }^{16}$ We next examine solitons in semiconductors when perturbations are present.

\subsection{Solitons with perturbations}

A variety of studies have shown that soliton evolution is altered by higher-order dispersion, loss, and nonlinear effects. ${ }^{17}$ Effects known from the study of glass optical fibers include third-order dispersion (TOD), Raman scattering, and self-steepening (SS), amongst others. ${ }^{18-20}$ In semiconductors, nonlinear absorption of multiple photons across the optical bandgap occurs when intense light is injected into the medium. This can take the form of two-photon absorption (TPA) or three-photon absorption (3PA), depending on the optical gap compared to the incident photon energy. In the case of silicon $\left(E_{g}=1.1 \mathrm{eV}\right) \mathrm{TPA}$ is the leading nonlinear loss, whereas for $\operatorname{GaInP}\left(E_{g}=1.9 \mathrm{eV}\right) 3 \mathrm{PA}$ is possible. The first impact of the nonlinear absorption is to reduce the desirable Kerr nonlinearity, requiring more power to counterbalance dispersion. Note that TPA requires much smaller threshold intensities compared to 3PA. Ultimately, both TPA and 3PA cap the peak power in the system, essentially removing a critical parameter to control the soliton dynamics and imposing an upper bound to the maximum compression achievable. The nonlinear absorption causes yet an additional higher-order effect in the form of free carriers which have their own dispersive (FCD) and absorptive (FCA) effects on the pulse. These free-carrier effects also interplay with and modulate the classical soliton evolution.

The illustrations in Fig. 2 provide an insight into the soliton regimes known in both glass and semiconductor media. In the canonical case, when only SPM and GVD are present, higher-order solitons are expected to exhibit periodic evolution along the propagation direction, and experience temporal compression at an early stage along the soliton period. ${ }^{14}$ At the point of maximum compression, shown as the narrow green pulse at the waveguide

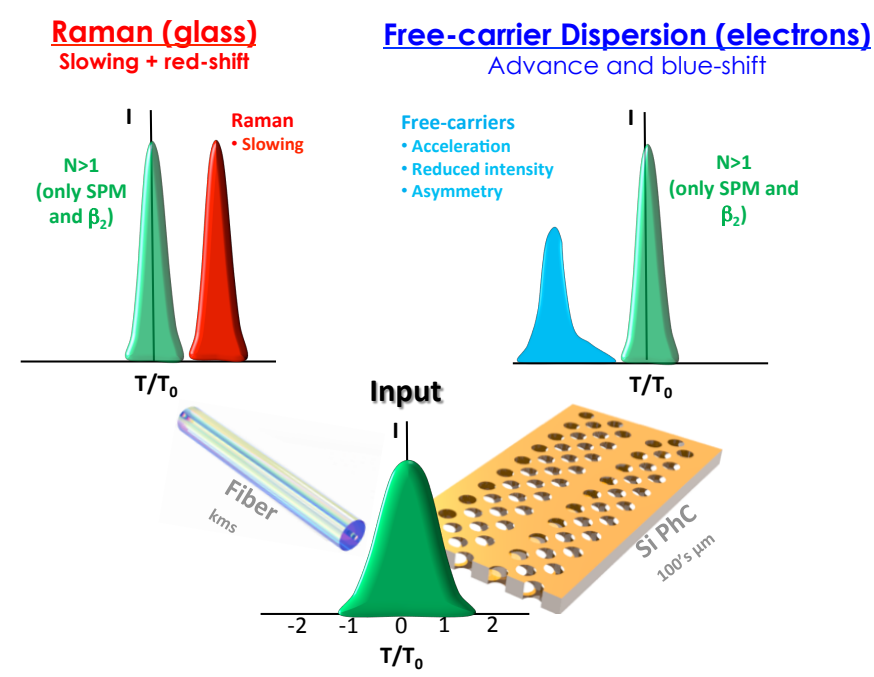

Figure 2. Schematic illustrating the differences between different soliton regimes. Time domain behavior of higher-order soliton compression in glass (Raman) and semiconductors (free carriers) with anomalous dispersion. 
output in Fig.2, the higher-order soliton is expected to exhibit higher intensity than the input pulse, with the same amount of energy contained in a shorter pulse. Nevertheless, aside from SPM and GVD, both in glass media and in semiconductors, higher-order effects, which can include multi-photon absorption, free carriers, or Raman, among others, alter the soliton evolution.

The left side of the figure describes intrapulse Raman scattering which plays the most important role among the higher-order effects in glass media. ${ }^{21}$ For ultrashort femtosecond pulses, the blue components of the pulse provide gain to the red components. As a consequence, the pulse red shifts, and for the short propagation lengths considered here, the amplitude of the soliton does not change. For longer lengths, the soliton will decay due to energy dissipation through Raman effects or linear loss. As bright solitons occur in media with anomalous dispersion, where low-frequency (red) components travel slower than high-frequency (blue) components, the Raman-induced red shift results in the pulse traveling more slowly. The right side of the figure describe intrapulse free-carrier effects typical of the temporal behavior in semiconductor waveguides. The output pulse exhibits asymmetry due to free-carrier dispersion (FCD) and absorption (FCA) and an intensity decrease due to nonlinear absorption. Owing to free-carrier frequency blue shift from the FCD, the pulse undergoes acceleration in the anomalous medium and arrives before the canonical soliton in time.

The presence and strength of these effects depends on the pulse duration. Whereas Raman processes typically require pulses in the femtosecond range for efficient intrapulse scattering, in contrast, free-carrier effects require longer pulses in time. Physically, this is explained by considering that the energy in the pulse tail needs to experience the free carriers generated by the leading edge in order to undergo a blue shift. Mathematically, the rate equation describing free-carrier generation involves an integral corresponding to the accumulation of free carriers across the pulse. ${ }^{22}$ Soliton-like propagation of femtosecond pulses in silicon channel waveguides has been observed through spectral measurements and cross-correlation, ${ }^{8}$ though no acceleration was observed as longer (picosecond) pulses are required for the non-instantaneous response of the free carriers to accumulate. We next describe experiments demonstrating temporal solitons in which the free-carriers play a significant role.

\subsection{Weak free carrier regime}

In our earlier experiments we used an optical autocorrelation measurement. While a good first approximation, we note that autocorrelations are by nature symmetric and require an approximation of the pulse shape to gain insight. As not all pulse dynamics are symmetric, information can be obscured and it is therefore desirable to obtain the real electric-field envelope of the optical pulses. With this motivation, we constructed a frequencyresolved optical gating (FROG) ${ }^{23}$ apparatus. FROG allows for simultaneous pulse intensity and phase retrieval in both the temporal and the spectral domains. We used the FROG to characterize pulse evolution in a GaInP waveguide (3PA limited) as a function of input pulse energy for a broad array of dispersion and nonlinear properties. Figure 3(a) are the retrieved temporal intensity (solid blue) and phase (dashed magenta) of the experimental FROG spectrograms for one particular case with retrieved optical gating errors less than 0.005 . We observe temporal narrowing characteristic of higher-order solitons, as well as the temporal separation indicating the onset of periodic evolution. However, as the FROG apparatus is after the chip, we only have access to the temporal shape, and not the time of flight information.

To understand the experiment better we conducted NLSE modeling of the experiment shown in the right panel. We notice a key point we couldn't discern from the FROG measurement. Namely, as we increase the pulse energy, the center of the pulse, defined by the first-order moment, forward shifts from 0 ps to -1.4 ps, indicating that the pulses advance in time while traveling along the slow-light photonic crystal. To discern the role of FCD in the temporal shift, the black dashed line indicates simulations with suppressed free-carrier effects $\left(N_{c}=0\right)$ at $19.4 \mathrm{pJ}(8.3 \mathrm{~W})$. The pulse center shifts noticeably less in this case, with a difference of 0.68 ps. We thus conclude that FCD is the origin of the soliton temporal advance and its accompanying blue-shift. We suspect the remaining shift might be due to strong self-steepening in the photonic crystal ${ }^{24}$ or residual pulse chirp. These initial results of solitons in the weak free-carrier regime (3PA generated) encouraged us to study this effect in the TPA-limited silicon material where we expect free carriers to play a more significant role.

\subsection{Strong free carrier regime}

Since TPA is of lower nonlinear order $\chi^{(3)}$, TPA-limited materials have a lower intensity threshold and experience larger attenuation and FCD compared to $\chi^{(5)} 3 \mathrm{PA}$-limited materials. ${ }^{25}$ Consequently, we expect free carriers 


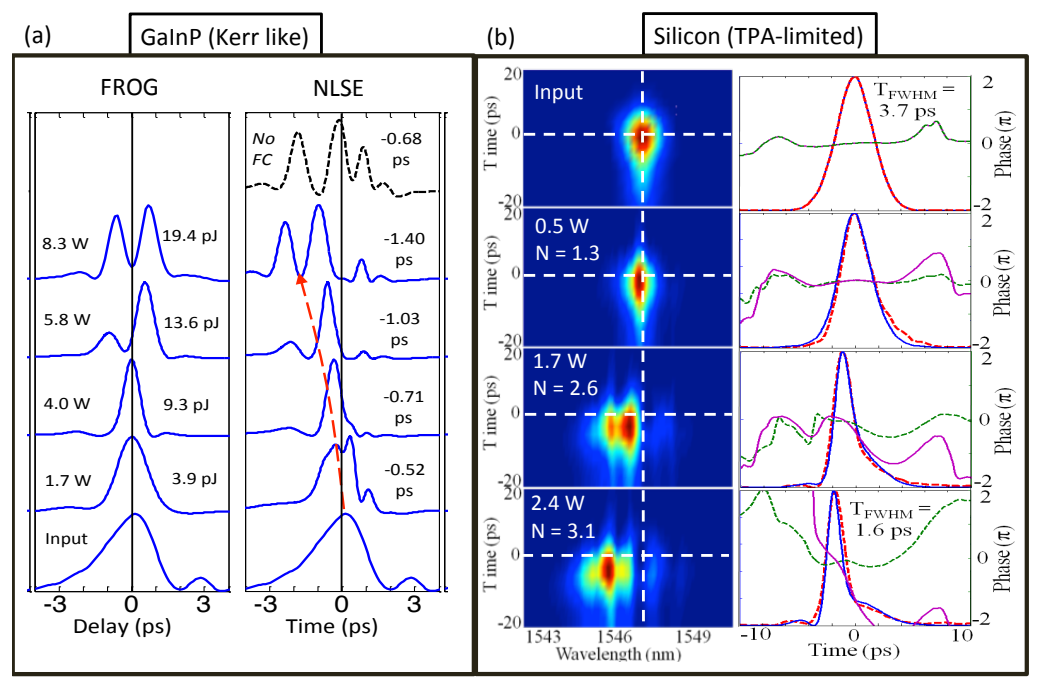

Figure 3. Soliton pulses with intrapulse plasma generation. (a) Weak free carrier regime in GaInP (Kerr $+3 \mathrm{PA})$. Experiment: FROG measurements after the waveguide. FROG retrieved time domain intensity (solid blue) as function of input power. On the right we show the NLSE modeled output corresponding to the FROG traces. The pulse temporally shifts to shorter delays with increased input power, as indicated by the temporal first-order moment (center of mass) of the pulses. The black dashed trace is a numerical simulation with suppressed free-carrier effects $\left(N_{c}=0\right)$, demonstrating the shift originates from the generation of a free-carrier plasma. The dashed red line acts a guide to the eyes to the original pulse center. Recall that FROG is relative time, e.g. $\tau=0$. (b) Strong free carrier regime in silicon (Kerr + TPA). Experiment: Cross-correlation FREG with time of flight. Left: Experimental FREG spectrograms. Right: Measured intensity (dashed red) and phase (dashed green) along with the NLSE-modeled intensity (blue) and phase (magenta) in the time domain following numerical deconvolution.

to play a more dominant role in the case of silicon. For this case, we have constructed an electrically gated apparatus (X-FREG) that is more more sensitive due to its linear detection scheme. ${ }^{26,27}$ We further implemented a cross-correlation configuration with which we also measure the pulse time of flight to obtain true time delay. Figure 3(b)(left) shows the FREG spectrograms for the input pulse and for the output pulse at three different coupled power levels for the silicon (TPA-limited experiment), along with the associated soliton number, $N$. The spectrograms clearly reveal the pulse spectrally blue-shifting with increasing power due to larger free-carrier concentrations. Further, we directly measure the time advance of pulses.

Figure 3(b)(right) shows the experimentally retrieved pulse intensities in the time domain indicated as the dashed red lines. We confirm these measurements with NLSE modeling (blue line), demonstrating excellent agreement. The effect of the free carriers over the trailing edge of the pulse noticeably results in the pulse developing a steep leading edge and getting advanced in time. We observe compression of 3.7-ps pulses to a minimum duration of $1.6 \mathrm{ps}$ (compression factor $\left.\chi_{c}=2.3\right)$ at $2.4 \mathrm{~W}(9 \mathrm{pJ})$. It is not obvious how much additional compression is possible in this system even with additional power due to the presence of TPA. It follows that there is likely an even lower scope for observing the characteristic temporal split and periodic recurrence. With further analysis of the data, we showed that the temporal advance here is greater than that in equivalent TPA-free media GaInP due to significantly stronger free-carrier effects in the silicon system. This makes sense given the free-carrier density observed in our silicon experiment $\left(N_{c} \sim 10^{17} \mathrm{~cm}^{3}\right)$ exceeds that observed in the TPA-free experiments ${ }^{11}$ by an order of magnitude at similar intensities, leading to much stronger interaction between the optical pulse and the free carriers in silicon.

In summary, we have shown time-resolved experiments demonstrating that soliton propagation dynamics in semiconductor media are governed by a complex nonlinear regime involving an intrapulse non-adiabatic freecarrier plasma (with absorptive and dispersive terms) generated from nonlinear absorption, giving rise to spectral blue shift and pulse temporal advance. 


\section{ACKNOWLEDGMENTS}

This research was supported by the Australian Research Council (ARC) Discovery Early Career Researcher Award (DECRA DE120102069), Center of Excellence CUDOS (CE110001018), ARC Laureate Fellowship (FL120100029), A.D.R, S.C., and acknowledge financial support from the ERC-Pharos programme lead by A. P. Mosk. T.F.K. was supported by EPSRC UK Silicon Photonics (Grant reference EP/F001428/1).

\section{REFERENCES}

[1] Agrawal, G. P., [Nonlinear Fiber Optics], Academic Press, fifth ed. (2014).

[2] Yin, L. and Agrawal, G. P., "Impact of two-photon absorption on self-phase modulation in silicon waveguides.," Optics Letters 32, 2031-3 (July 2007).

[3] Wood, W., Siders, C., and Downer, M., "Measurement of femtosecond ionization dynamics of atmospheric density gases by spectral blueshifting.," Physical Review Letters 67, 3523-3526 (Dec. 1991).

[4] Rieger, G. W., Virk, K. S., and Young, J. F., "Nonlinear propagation of ultrafast 1.5 um pulses in highindex-contrast silicon-on-insulator waveguides," Applied Physics Letters 84, 900-902 (2004).

[5] Fedotov, A. B., Serebryannikov, E. E., and Zheltikov, A. M., "Ionization-induced blueshift of high-peakpower guided-wave ultrashort laser pulses in hollow-core photonic-crystal fibers," Physical Review A 76(5), 053811 (2007).

[6] Blanco-Redondo, A., Eades, D., Li, J., Krauss, T. F., Eggleton, B. J., and Husko, C., "Controlling freecarrier temporal effects in silicon by dispersion engineering," Optica 1, 299-306 (2014).

[7] Colman, P., Husko, C., Combrié, S., Sagnes, I., Wong, C. W., and De Rossi, A., "Temporal solitons and pulse compression in photonic crystal waveguides," Nature Photonics 4, 862-868 (Nov. 2010).

[8] Ding, W., Gorbach, A. V., Wadswarth, W. J., Knight, J. C., Skryabin, D. V., Strain, M. J., Sorel, M., and De La Rue, R. M., "Time and frequency domain measurements of solitons in subwavelength silicon waveguides using a cross-correlation technique.," Optics Express 18, 26625-30 (Dec. 2010).

[9] Travers, J. C., Chang, W., Nold, J., Joly, N. Y., and St. J. Russell, P., "Ultrafast nonlinear optics in gas-filled hollow-core photonic crystal fibers," Journal of the Optical Society of America B 28(12), A11 (2011).

[10] Saleh, M., Chang, W., Hölzer, P., Nazarkin, A., Travers, J., Joly, N., Russell, P., and Biancalana, F., "Theory of Photoionization-Induced Blueshift of Ultrashort Solitons in Gas-Filled Hollow-Core Photonic Crystal Fibers," Physical Review Letters 107, 203902 (Nov. 2011).

[11] Husko, C., Combrié, S., Colman, P., Zheng, J., De Rossi, A., and Wong, C. W., "Soliton dynamics in the multiphoton plasma regime.," Scientific Reports 3, 1100 (Jan. 2013).

[12] Blanco-Redondo, A., Husko, C., Eades, D., Zhang, Y., Li, J., Krauss, T. F., and Eggleton, B. J., "Observation of soliton compression in silicon photonic crystals," Nature Communications 5, 3160 (Jan. 2014).

[13] Bhat, N. A. R. and Sipe, J. E., "Optical pulse propagation in nonlinear photonic crystals," Physical Review E 64(5), 056604 (2001).

[14] Mollenauer, L. F., Stolen, R. H., and Gordon, J. P., "Experimental observation of picosecond pulse narrowing and solitons in optical fibers," Physical Review Letters 45(13), 1095-1098 (1980).

[15] Li, J., White, T. P., O'Faolain, L., Gomez-Iglesias, A., and Krauss, T. F., "Systematic design of flat band slow light in photonic crystal waveguides.," Optics Express 16, 6227-32 (Apr. 2008).

[16] Combrié, S., Tran, Q. V., De Rossi, A., Husko, C., and Colman, P., "High quality GaInP nonlinear photonic crystals with minimized nonlinear absorption," Applied Physics Letters 95, 221108 (2009).

[17] Dudley, J. M., Genty, G., and Coen, S., "Supercontinuum generation in photonic crystal fiber," Reviews of Modern Physics 78, 1135-1184 (Oct. 2006).

[18] Golovchenko, E. A., Dianov, E. M., Prokhorov, A. M., and Serkin, V. N., "Decay of optical solitons," Journal of Experimental and Theoretical Physics Letters 42(2), 87-91 (1985).

[19] Wai, P. K., Menyuk, C. R., Lee, Y. C., and Chen, H. H., "Nonlinear pulse propagation in the neighborhood of the zero-dispersion wavelength of monomode optical fibers.," Optics Letters 11, 464-6 (July 1986).

[20] Tai, K., Hasegawa, A., and Bekki, N., "Fission of optical solitons induced by stimulated Raman effect," Optics letters 13, 392 (1988).

[21] Gordon, J. P., "Theory of the soliton self-frequency shift.," Optics Letters 11, 662-664 (1986). 
[22] Lefrancois, S., Husko, C., Blanco-Redondo, A., and Eggleton, B. J., "Nonlinear silicon photonics analyzed with the moment method," Journal of the Optical Society of America B 32(2), 218 (2015).

[23] Trebino, R., [Frequency-Resolved Optical Gating: The Measurement of Ultrashort Laser Pulses], Springer (2000).

[24] Husko, C. and Colman, P., "Giant anomalous self-steepening in photonic crystal waveguides," Physical Review A 92, 013816 (2015).

[25] Husko, C., Combrié, S., Tran, Q. V., Raineri, F., Wong, C. W., and De Rossi, A., "Non-trivial scaling of selfphase modulation and three-photon absorption in III-V photonic crystal waveguides," Optics Express 17, 22442 (Nov. 2009).

[26] Dorrer, C. and Kang, I., "Simultaneous temporal characterization of telecommunication optical pulses and modulators by use of spectrograms.," Optics Letters 27, 1315-7 (Aug. 2002).

[27] Thomsen, B., Roelens, M., Watts, R., and Richardson, D., "Comparison between nonlinear and linear spectrographic techniques for the complete characterization of high bit-rate pulses used in optical communications," IEEE Photonics Technology Letters 17, 1914-1916 (Sept. 2005). 